\title{
REPRODUCCIÓN DE LAS SOCIEDADES RURALES (1)
}

\author{
Nelly del Carmen Suárez R. ${ }^{\text {i }}$ \\ ${ }^{\text {i }}$ Filósofa, M.s.c. en Desarrollo Comunitario y Educación de Adultos, Esp. en \\ Planeamiento educativo. Profesora Titular de la Universidad de Caldas adscrita al \\ departamento de Desarrollo Rural de la Facultad de Ciencias Agropecuarias.
}

Manizales, 2008-05-05 (Rev. 2008-06-12)

\section{RESUMEN}

En su afán por crear las condiciones existenciales necesarias para alcanzar logros de vida acordes con las situaciones sociales actuales, los diversos grupos humanos, entre éstos los familiares, se ingenian diversas maneras para superar las adversidades. Estas maneras son denominadas por unos como estrategias de supervivencia y por otros como medios de vida. La primera es un marco explicativo que se apoya en la presunción de que las familias manejan un margen de libertad a partir del cual pueden formular como grupo sus propios cursos de acción -estrategias- incluso por encima de los que formulan sus miembros individualmente. La segunda se soporta en el enfoque Medios de Vida según el cual las familias toman decisiones y actúan a partir del conjunto de capacidades, activos (recursos tanto materiales como sociales y culturales) y actividades con que cuentan. El presente documento aborda de manera analítica estas dos propuestas.

\section{PALABRAS CLAVE}

Sociedades rurales, medios de vida, estrategias familiares.

\section{REPRODUCTION OF RURAL SOCIETIES}

\begin{abstract}
In their quest to create the conditions necessary to obtain life achievements in agreement with the current social situations, different human groups have come up with various ways to overcome adversity. These ways are called by some survival strategies and by others livelihoods. The first is an explanatory framework that is based on the assumption that families handle a margin of freedom from which they can, as a group, construct their own courses of action - strategies - even over those formulated individually by its members. The second approach is supported by the Livelihoods focus whereby families make decisions and act based on the set of capacities, assets (material, social and cultural resources) and activities available to them. This document analytically addresses these two proposals.
\end{abstract}




\section{KEY WORDS}

Rural societies, livelihoods, family strategies.

\section{Presentación}

Los esfuerzos por comprender y explicar los procesos y las dinámicas mediante las cuales las sociedades rurales se re-producen, se manifiestan en varias propuestas teóricas y metodológicas. Uno de los centros de atención de estas propuestas lo constituyen las múltiples y diversas maneras creativas como históricamente las familias rurales han respondido ante las presiones y las oportunidades que les brinda el mundo socio-cultural y natural.

Este documento recoge, de manera general, dos de las principales propuestas puestas en práctica en América Latina, cada una de éstas con sus respectivos planteamientos teóricos y metodológicos: una mirada histórica, Estrategias familiares, Medios de vida.

\section{Una mirada histórica}

Los ordenamientos sociales en América Latina, particularmente, han promovido la existencia de grupos humanos que se ven obligados a realizar su vida en condiciones sociales, económicas, políticas, culturales y medio-ambientales, desventajosas. En su lucha por supervivir, estos grupos toman decisiones y planean actuaciones que les permiten no sólo superar las limitaciones existenciales creadas por la sociedad a la que pertenecen sino también aportar nuevas dinámicas y estilos de vida.

En la década de los setenta, incursionó en los discursos de académicos, instituciones y políticos del desarrollo, el concepto de estrategias de supervivencia. Este concepto se aplicaba a un conjunto de actividades realizadas por los sectores populares de América Latina para hacer frente a sus carencias existenciales, se aplicaba sobre todo a actividades relacionadas con el comercio informal considerado como la principal manifestación de dichas estrategias.

A comienzos de los años ochentas, Argueyo (2) define las estrategias de supervivencia como "el conjunto de acciones económicas, sociales, culturales y demográficas que realizan los estratos poblacionales que no poseen medios de producción suficientes ni se incorporan plenamente al mercado, por lo que no obtienen de los mismos sus ingresos regulares para mantener su existencia en el nivel socialmente determinado, dadas las insuficiencias estructurales del estilo de desarrollo dominante".

Las ocupaciones generadas en el sector informal de la economía fueron algunas de las principales estrategias a las que tuvieron que recurrir los pobladores de los sectores populares para: a) hacerle frente a la incapacidad estatal para satisfacer las aspiraciones más elementales de los pobres (3) y b) como un instrumento de empleo para las personas más pobres, con menores niveles de escolaridad y baja calificación laboral (4)

Félix Acosta (5) reconoce en México una corriente de investigación que opta por el concepto de estrategias de reproducción apoyada en los planteamientos hechos por 
Chayanov en torno a la unidad doméstica campesina como una unidad económica de producción y consumo. Esta unidad permite vincular las actividades de producción y consumo y analizar las interrelaciones entre el grupo familiar y la unidad productiva. En este contexto, las estrategias de reproducción se refieren a "un conjunto de acciones orientadas por motivos conscientes o no, desplegadas por las familias para garantizar su supervivencia”, (6).

\section{Estrategias familiares}

En la actualidad, Garrido y Gil (7), dos académicos españoles, proponen unos elementos teórico-conceptuales para explicar y orientar maneras de abordar analíticamente las formas de reproducción de las familias. Plantean que el término estrategia se usa, de manera general, para referirse "a toda selección de cursos alternativos de acción (recursos tácticos), por su virtualidad para producir resultados futuros (objetivos estratégicos) en situaciones de incertidumbre"(8).

De acuerdo con estos autores, la anterior definición está estructurada a partir de tres aspectos básicos: a) un margen de maniobra o existencia de un abanico de posibilidades de elección; b) unos objetivos a largo plazo previamente definidos; y c) la existencia de alguna clase de incertidumbre en el entorno, de manera que haya una acción estratégica y no sólo un acto reflejo. Como los sujetos sociales actúan siempre en entornos poblados por otros sujetos también dotados de recursos tácticos, esta capacidad estratégica puede ser interferida por lo impredecible de los comportamientos de esos otros sujetos e incluso de los parámetros o características del entorno.

Aplicada a la vida en familia, el término estrategia da cuenta de "las asignaciones de recursos humanos y materiales, a actividades realizadas por un grupo particular de personas relacionadas entre si por parentesco (consanguíneo o afin), con el objeto de maximizar su aptitud para adaptarse a entornos materiales y sociales"(9).

Según Garrido y Gil:

- Los recursos tácticos de las familias están representados por: a) sus propios miembros, en tanto son quienes activan las estrategias actuando y decidiendo sobre la composición, organización y estructura de la familia, b) los bienes que controlan y c) las actividades que pueden realizar.

- Los objetivos se representan en un mayor y mejor éxito en su adaptación al entorno. En la práctica, las familias se orientan más por experiencias previas, es decir, por el éxito de los resultados anteriormente producidos en su proceso de adaptación al entorno, que por intencionalidades expresadas por los miembros de las familias.

- Los entornos social y natural contienen a las demás familias (con las que contraen diversos vínculos), a las organizaciones e instituciones con las cuales interactúan permanentemente y al ambiente natural.

El núcleo de estas estrategias descansa sobre el margen de variación que tienen las acciones o sobre las maniobras -recursos tácticos- que las familias realizan respondiendo a su condición de grupo. Lo anterior significa que estas estrategias son 
posibles gracias a la elasticidad con que los diversos miembros de las familias adaptan sus comportamientos a los vaivenes y a los cambios del entorno material y social (10).

La variación o elasticidad de las acciones familiares está sujeta a determinaciones históricas y su presencia se explica por el carácter de constructo social que tiene la realidad humana en general y la familiar en particular y, por consiguiente, todas sus dimensiones y componentes. Al ser parte del proceso de construcción social, estas realidades son indeterminadas, es decir, nunca están concluidas ni finalizadas sino siempre en proyecto, en posibilidad.

La propuesta de las estrategias familiares como marco explicativo de esta dinámica social, suele apoyarse en la presunción de que las familias manejan un margen de libertad a partir del cual pueden formular como grupo sus propios cursos de acción estrategias- incluso por encima de los que formulan sus miembros individualmente. Esta posibilidad se da sobre todo cuando hay un patrimonio que determina en buena medida: a) el funcionamiento de la familia como una unidad colectiva y b) el comportamiento de cada uno de sus miembros teniendo en cuenta los requerimientos del grupo como tal $\underline{(11)}$.

En este sentido, "todas las familias por muy constreñidas que estén por la necesidad física, disponen de recursos y alternativas que administran con su mejor voluntad para sacar partido a la escasez. Y en tanto que lo hagan están adoptando estrategias” (12) .

Lo anterior no niega ni contradice la existencia de limitaciones objetivas estructurales tanto naturales como sociales que tienden a constreñir el margen de acción y de elección de las familias. Por ejemplo, en algunas sociedades hay ciertas formas de organización que limitan en las familias su acceso y control a recursos de subsistencia, imponiéndole restricciones a las parejas en cuanto al número de hijos deseados. De esta manera presionan hacia modelos de familia basados en la primogenitura; sin embargo, son las mismas parejas quienes finalmente toman la decisión, haciendo uso de su margen de libertad. Este margen de libertad abre la posibilidad a las estrategias -conjunto de acciones y decisiones que toman las familias-.

En 1985 Nhora Cebotarev (13) propone el concepto de "indeterminación familiar" para dar cuenta de ese margen de libertad que tienen las familias para asumir las estructuras propias y las de la sociedad bien sea como "dadas" -en este caso sin posibilidad de escapar a su influencia- o como resultados históricos de acciones humanas -sujetas, por tanto, a nuevos replanteamientos- cuya modificación implica trascender patrones culturales e influencias tradicionales.

La aceptación del contexto de indeterminación en el que se mueven las familias conlleva a reconocer que este grupo social no es un ente pasivo sino un sujeto activo que constantemente, y de manera consciente y crítica, está haciendo análisis tanto de las presiones que sobre ella genera el medio socio-natural como de las necesidades y las expectativas de cada uno de sus miembros. Las familias utilizan estos análisis para tomar decisiones que les permitan colmar sus aspiraciones respecto a estilos de vida y a comportamientos deseados, también para controlar y/o permear las influencias que les llega desde afuera, muchas veces con tal fuerza que sus acciones se convierten en una presión capaz de promover cambios estructurales a nivel de la sociedad. 
En las ciencias sociales el concepto estrategia se torna en una perspectiva que permite abordar de manera analítica y comprensiva la realidad familiar. En tanto tal, tiene la capacidad de ofrecer puentes interdisciplinarios que sintetizan perspectivas aparentemente contradictorias y excluyentes $\underline{(14)}$ :

- Entre el subjetivismo y el objetivismo cuando se dan a la tarea de brindar explicaciones de los fenómenos sociales. Tanto en la historia social como en la historia de la familia ha estado presente una perspectiva dicotómica entre las mentalidades y las estructuras. La perspectiva de las estrategias correlaciona la búsqueda de objetivos a largo plazo (asociado a las mentalidades) con recursos tácticos de que se disponen en el plano de las familias.

- En la consideración de los actores sociales, las familias se pueden considerar bien sea como objetos-receptoras de cambios -perspectiva determinista y estática- o como sujetos-agentes de cambio social -perspectiva voluntarista e historicista-. En este caso, la familia es un actor-autor de su propio destino que actúa en un contexto de determinación parcial.

- En la conocida oposición entre la perspectiva micro -que reduce las organizaciones y fenómenos sociales a sus componentes más elementales y, en último término, a relaciones interpersonales- y la perspectiva macro -que ignora las singularidades locales y sólo toma en cuenta las regularidades estadísticas. Según la perspectiva de las estrategias, en el manejo del margen de libertad hay un inter-juego de fuerzas micro/macro.

- $\mathrm{Su}$ principal virtud es superar ampliamente la capacidad explicativa del paradigma dominante de las funciones de la familia consagrado por la sociología ortodoxa de la familia que fundamenta la existencia de este grupo social incluso imponiendo una determinada forma- por su funcionalidad al sistema macrosocial. Lo central para la perspectiva de las estrategias son las actuaciones de las familias dentro de márgenes de libertad teniendo como referente la misma familia y sus miembros.

\section{Medios de vida (15)}

Es una propuesta sustentada en el enfoque Medios de Vida elaborado por Chambers y Conway. Comprende el conjunto de capacidades, activos (recursos tanto materiales como sociales y culturales) y actividades con que cuentan las familias en términos de medios de vida (16). En esta definición se pone el acento en la relación entre los activos y las opciones que, en la práctica, poseen estos grupos sociales para buscar y realizar actividades alternativas que puedan generarles resultados en el nivel necesario y esperado para supervivir.

En el ámbito internacional del desarrollo, la búsqueda a nivel conceptual y práctico de respuestas que contribuyeran a erradicar la pobreza en el mundo, sirvió como caldo de cultivo para la emergencia del enfoque Estrategias de Vida Sostenibles propuesto y utilizado por el Departamento de Desarrollo Internacional del Reino Unido (Department for Internacional Development, DFID). Este departamento reconoce que si bien la teoría de los medios de vida fue planteada y desarrollada por Robert Chambers durante los 
años 80 y comienzos de los 90, actualmente representa un nuevo punto de partida desde un punto de vista político y práctico (17).

Dietmar Stoian y Jason Donovan (18), apoyados en Chambers y Conway (1991), Bebbington (1999), DFID (1999), ubican el surgimiento de este enfoque a finales de la década de los 80 como respuesta a la necesidad de contar con un marco propicio para el análisis integral de las estrategias de vida de los hogares rurales. Resaltan el hecho de que a diferencia de otros enfoques convencionales que se concentran, a menudo, en los sistemas productivos o fincas, el enfoque de medios de vida parte del hogar como unidad socioeconómica y analítica dotada con cinco formas de capital, medios de vida o activos, propuestos por el DFID británico en 1999:

- Capital humano; aptitudes, conocimientos, capacidades laborales y buena salud.

- Capital social: redes y conexiones, participación en grupos formales (adhesión a reglas, normas y sanciones acordadas de forma mutua y comúnmente aceptadas, relaciones de confianza, reciprocidad e intercambios).

- Capital natural: acceso y calidad de recursos naturales (agua, tierra, árboles, animales).

- Capital físico: infraestructuras básicas y bienes de producción (red vial y medios de transporte, comunicaciones, suministro de agua y energía, edificios, equipos $\mathrm{y}$ herramientas).

- Capital financiero: disponibilidad de dinero (ahorros, créditos, pensiones, remesas).

Una de las particularidades de este enfoque consiste en que no considera a los activos como rígidos ni excluyentes entre sí, pues en caso de la ausencia o debilidad de uno de ellos, éste puede ser sustituido por otro. Deja en claro que aunque los activos son parte de las estrategias de vida de las familias, su utilización o creación está influenciada por: a) las preferencias y prioridades de cada grupo familiar en particular, b) algún tipo de vulnerabilidad como desastres naturales, escasez de recursos, estacionalidad y, c) las estructuras y procesos gubernamentales y privados entre los cuales se consideran los factores institucionales y culturales y las políticas públicas.

En los fundamentos del enfoque está una crítica al modelo de ingreso y consumo utilizado comúnmente para evaluar la pobreza, según este modelo, una persona es pobre cuando su nivel de ingreso se encuentra bajo una línea de pobreza previamente definida, o si su consumo está por debajo de un mínimo estipulado. El enfoque Medios de Vida, por su parte, considera que la pobreza tiene que ver con, además del ingreso, la ausencia de capacidades básicas para satisfacer necesidades físicas, emocionales y sociales y para alcanzar objetivos de participación en la vida de las comunidades e influir en la toma de decisiones.

Los grupos familiares realizan al mismo tiempo múltiples actividades como parte de sus estrategias de vida (19), en este sentido pueden combinar, por ejemplo, la explotación agrícola en el propio predio con la venta de mano de obra familiar a nivel local y/o con la migración de alguno de sus miembros. En este mismo sentido, los resultados que esperan obtener también son múltiples y diversos: éstos van desde la creación o apropiación de mecanismos participativos que les permitan llegar a opinar en un escenario de discusión comunitario hasta el incremento de ingresos pasando por el desarrollo de un sentimiento de seguridad existencial, entre otros. Estos resultados, en 
muchos casos, ni siquiera son tangibles. Entre los resultados esperados se encuentran: mayor bienestar, menor vulnerabilidad, mayor seguridad alimentaria, usos más sostenibles de la base de recursos naturales. (20)

John Farrington identifica los siguientes principios básicos del enfoque estrategias de vida $(21)$ :

- Focalización en las personas, así sea que la estrategia se ubique a nivel macro (contexto de la sociedad), a nivel meso (contexto de la comunidad o la localidad) o a nivel micro (contexto de las familias). Su aplicación empieza con un análisis de las estrategias de vida de las personas y de la forma en que han cambiado con el tiempo. Involucra a las personas y las ayuda a lograr sus propios objetivos de vida. Se centra en el impacto que ejercen las políticas y disposiciones institucionales sobre ellas y busca incidir en éstas de manera que promuevan la agenda en beneficio de los pobres.

- Holismo: no es sectorial y se puede aplicar a todos los grupos sociales. Reconoce la diversidad presente en las estrategias de vida que adoptan las personas, los múltiples actores que intervienen en este proceso, la variedad de influencias que reciben las personas, y busca establecer relaciones entre ellas. Pretende múltiples resultados determinados y negociados por las propias personas.

- Establecimiento de vínculos a nivel micro y macro: Como los activos y aspiraciones de las personas y de los grupos son los pilares del enfoque, los esfuerzos por reunir información acerca de éstos tienen una orientación micro pero muchos de los factores que afectan las estrategias tienen características de nivel macro. Por ejemplo, el acceso a capital financiero puede depender de políticas relativas al sector financiero y al sector bancario rural en general.

- Sostenibilidad y compensación: Se relaciona tanto con la reserva de los recursos naturales como con las personas y las instituciones que respaldan las actividades que hacen parte de sus estrategias de vida. La compensación se refiere a la posibilidad de sustituir un tipo de capital por otro teniendo en cuenta que la factibilidad o aceptabilidad de estos intercambios de capitales depende del contexto en el cual viven las personas y los grupos.

Desde un punto de vista analítico este enfoque es apto para ser ejecutado especialmente a nivel de familia, de hogar o de familia/hogar y, de manera agregada, a nivel de la comunidad o del territorio. En este caso permite analizar:

a) La combinación y recombinación de actividades productivas que tienen un carácter de subsistencia con actividades comerciales.

b) La influencia que las estructuras y procesos institucionales en niveles meso y macro, ejercen sobre estas actividades.

c) La posible combinación de la producción de subsistencia con otras actividades que generen ingresos.

d) La determinación de la importancia de la producción de subsistencia para la seguridad alimentaria de las familias rurales. 
Desde un punto de vista práctico, el estudio de los medios de vida se torna complicado debido al dinamismo y a la variabilidad presentes en ellos. Fundamentalmente la permanente y diversa combinación de actividades que realizan las familias rurales, los asuntos de género relacionados con la distribución de tareas y la apropiación de ingresos entre hombres y mujeres, son objeto de cambios rápidos durante todo el tiempo.

Desde una perspectiva de género, con énfasis en mujer, el enfoque hace esfuerzos por saldar la deuda histórica que las sociedades han contraído con este grupo social, especialmente con las mujeres rurales. En este sentido, Ellis (22) resalta la necesidad de reconocer, en los estudios empíricos en los que se aplique el enfoque medios de vida, las desventajas sociales, políticas, legales, económicas y laborales que tienen las mujeres rurales respecto de los hombres. Estas desventajas refuerzan las dependencias que tienen las mujeres de los hombres y les recorta, en el plano individual y en el familiar, posibilidades de tomar decisiones relacionadas con estrategias de vida que les mejore sus condiciones existenciales como mujeres. Esta limitación cobra fuerza en mujeres que están al frente de un grupo familiar.

Para el caso de las mujeres, la diversificación presente en las estrategias de vida de las familias puede reforzar, más que reducir, las condiciones que provocan inequidades de género, mientras a los hombres les puede permitir disfrutar de nuevas libertades y expandir sus elecciones cuando están construyendo sus estrategias de vida individual y familiar. Esta diversificación puede estar condenando a las mujeres a vivir una existencia de esclavitud alrededor y a partir de una variedad de tareas $y$ responsabilidades que, incluso, se superponen en su realización día a día.

\section{Posibilidades y limitaciones del enfoque Estrategias de Vida}

Quienes defienden el enfoque de Estrategias de Vida (llamado también, Modos de Vida Sostenible o Medios de Vida Sostenible) como una alternativa viable, creíble y prometedora para programar acciones orientadas a reducir la pobreza, se sustentan en los siguientes argumentos:

- El enfoque no es una receta que se pone en acción de manera repetitiva y homogénea. Es un esfuerzo por intervenir en el medio rural con propósitos de cambio positivo, después de haber comprendido, previo un estudio empírico, las diversas maneras como las familias rurales organizan sus vidas a través de un proceso de interacción creadora con el entorno social, político, económico y natural.

- Es una forma de acercarse a lo rural desde el pensar la vida de las personas.

- En este sentido es innovador y se orienta por el reconocimiento de la complejidad de la vida humana en los niveles individual, familiar y social.

- Es una alternativa teórica y metodológica que permite realizar análisis más integrales y reales de las vidas de las personas y de los grupos familiares, desde los cuales es posible soportar y acompañar propuestas de desarrollo más 
significativas socialmente, pertinentes culturalmente y efectivas en términos de acceso y mejoramiento de activos o recursos.

- Al asumir como objetivo central el mejoramiento de las estrategias (activos o recursos) de vida de las familias, da a entender que el proceso no va a ser invasivo, pues de lo que se trata es de involucrarse en la dinámica misma de los grupos familiares y de las comunidades reconociendo y respetando sus particularidades, valorando sus capacidades y habilidades, sus saberes cotidianos y sus prácticas de vida como fuentes y fundamento de una organización social; pero, además, como bases de una propuesta orientada a lograr mejores formas y niveles de vida.

- Abre nuevas perspectivas de trabajo al centrarse en lo que la gente hace y en las decisiones que toma alrededor del uso de sus recursos o activos (de infraestructura, económicos, de producción $\mathrm{y}$ naturales), de sus propias capacidades y posibilidades y de lo que le ofrece el entorno político e institucional.

- El hecho de concentrarse en la familia permite considerar que finalmente se reconoce y da la importancia merecida a este grupo social que si bien está presente en los discursos de los distintos actores y sectores sociales, realmente no hacen parte estructural ni de las políticas ni de las prácticas del desarrollo.

\section{Crítica}

El análisis de algunas experiencias en las que se ha aplicado este enfoque (23) deja al descubierto que no facilita necesariamente un cambio de la situación de pobreza en que se encuentran millares de familias productoras rurales. Esta limitación del enfoque se debe al hecho de que en el mundo real estas familias no sólo son objeto de una marginalización política sino que también sufren los efectos del establecimiento y ejercicio de unas relaciones sociales por parte de una minoría que distorsiona el manejo del poder con el fin de acumular activos restringiéndoles a otros su acceso a éstos.

De acuerdo con lo anterior, valdría la pena incorporar entre los activos o recursos el capital político. Para elaborar sus estrategias de vida, las familias rurales requieren ser habilitadas por las sociedades respectivas para determinar su propio futuro y contar con las estructuras y los procedimientos políticos necesarios para garantizarlo (24) .

El hecho de estar amarrado desde su gestación a la superación de la pobreza, no sólo lo restringe en su concepción y aplicación sino que le resta a la posibilidad de articularse o elevarse a la categoría de propuesta alternativa de desarrollo rural. Los asuntos de género, a pesar de estar presente en los principios enunciados en el enfoque de medios de vida, aún siguen siendo débiles, cuando no invisibles, tanto a las miradas teóricas como en los procesos y prácticas del desarrollo basadas en este enfoque.

Finalmente, y desde una mirada más general al concepto de estrategias aplicadas a la población rural, autores como Jorge Mercado Mondragón (25) se preguntan si aún es válida la pertinencia del concepto. Particularmente este estudioso considera que en el momento actual las estrategias de reproducción a las que están recurriendo las familias campesinas no responden a sus dinámicas y lógicas de vida sino a fenómenos 
provocados por la política de ajuste estructural aplicados a los países llamados en vía de desarrollo a partir de las dos últimas décadas del siglo pasado:

"En efecto, no creemos que la puesta en marcha de las políticas neoliberales hayan permitido desarrollar en el seno de las comunidades campesinas estrategias de reproducción en el sentido de una lógica y una racionalidad perfectamente definidas. Un concepto de esa naturaleza supone la existencia de acciones concretas de parte del campesino que son elaboradas y analizadas para ser finalmente llevadas a cabo".

Mercado Mondragón propone la utilización del término dinámicas familiares rurales para la reproducción porque permite medir los cambios que se están operando en el seno de las unidades de producción familiar campesinas, y hacer análisis de la adaptación, del cambio de lógica y de racionalidad campesina, lo cual se manifiesta a través de fenómenos crecientes como son la venta de la tierra, la substitución del trabajo agrícola por el empleo no agrícola y la migración -que de individual se convierte día con día en familiar- y de temporal pasa a ser permanente.

\section{BIBLIOGRAFÍA}

- Acosta, Félix. La familia en los estudios de población en América Latina: Estado del conocimiento y necesidades de investigación. Universidad Autónoma del Estado de México. Toluca, México. Revista electrónica Papeles de Población, Julio/septiembre. 2003. http://www.redalyc.uaemex.mx/redalyc/src/inicio/artpdfred.jsp?!CveNumRev=7 76\&iCveEntRev=112.

- Acosta, Luis Alejandro y Rodríguez, Marcos Sebastián. En busca de la agricultura familiar en América latina. En línea:

- Oficina Regional de la FAO para América latina. www.rlc.fao.org/prior/desrural/pdf/busca.pdf -

- Argueyo (1982) citado por Sanchez, J. estrategias de supervivencia en la comunidad andina. Centro Andino de Acción Popular. Quito, 1984

- Chambers, Robert and Conway, R. 1992. Sustainable Rural Livelihoods: Practical Concepts for the 21st Century. IDS Discussion Paper, No 296, p. 7

- Durston, John. Juventud y Desarrollo Rural. Marco conceptual y contextual. COMISION ECONOMICA PARA AMERICA LATINA Y EL CARIBE CEPAL. 1998. Serie Políticas Sociales 28. Santiago de Chile

- De Soto, Fernando. El otro sendero. La revolución informal. Oveja Negra. Bogotá, 1987

- Ellis, Frank. 2000. Rural livelihoods and Diversity in developing Countries. Oxford, University Press. New York.

- FARRINGTON JOHN, CARNEY DIANA, ASHLEY CAROLINE Y TURTON CATHRYN. Estrategias de vida sostenibles en la práctica: Primeras aplicaciones de los conceptos en áreas rurales. Revista No 42. Junio 1999. http://www.rimisp.cl/www.oneworld.org/odi.

- González Rodríguez Juan José. Efectos perversos de las estrategias familiares en la agricultura. En: Luis Garrido Medina y Enrique Gil Calvo (eds). Estrategias Familiares. Madrid: Alianza Universidad, 1993. P. 134. 
- GRAN BRETAÑA. DEPARTMENT FOR INTERNATIONAL DEVELOPMENT DFID. Hojas orientativas sobre los medios de vida sostenibles. Conceptos Básicos. (On line). (Citado 17 octubre de 2005). Disponible en http:/www.

- Mercado Mondragón Jorge. El concepto dinámicas familiares rurales de producción y la pertinencia de su aplicación en la sociología rural. VII Congreso Latinoamericano de Desarrollo Rural. 20-24 de noviembre de 2006. Quito, Ecuador. www.alasru.org/cdalasru2006/22\%20GT\%20Jorge\%20Mercado\%20Mondragón .pdf. Consultado el 24 de mayo de 2007

- Quejandría Benjamín, Monares, Aníbal y Ugarte de Peña Montenegro Raquel. Hacia una región sin pobres rurales. FONDO INTERNACIONAL DE DESARROLLO AGRICOLA FIDA, División América Latina y el Caribe. Agosto 2003, Santiago de Chile. http://www.ifad.org/operations.regional/2002/pl/regionalstrategy s.pdf

- Stoian Dietmar, Donovan Jason. Articulación del mundo campesino con el mercado. Integración de los enfoques medios de vida y cadena productiva. VI semana científica del CATIE.

\section{NOTAS}

1. Este documento hace parte del contexto teórico elaborado por la autora para la investigación "Dinámicas de vida en familias rurales. Caso de la Vereda San Peregrino del municipio de Manizales, Caldas".

2. Argueyo (1982) citado por Sanchez, J. Estrategias de supervivencia en la comunidad andina. Centro Andino de Acción Popular. Quito, 1984.

3. De Soto, Fernando. El otro sendero. La revolución informal. Oveja Negra. Bogotá, 1987.

4. Londoño, J. Evolución reciente del empleo y el desempleo urbano. En: revista Economía Colombiana. Bogotá, 1985.

5. Acosta, Félix. Opus cit.

6. Ibíd.

7. Este aparte es una adaptación de los planteamientos hechos por Luis Garrido Medina y Enrique Gil Calvo en su artículo: El concepto de estrategias familiares. En: Garrido Medina, Luis y Gil Calvo, Enrique (eds). Estrategias Familiares. Madrid: Alianza Universidad, 1993. P. 13-36.

8. Ibíd. p. 14.

9. Ibíd. p. 15.

10. Según Garrido y Gil, op cit, p. 15, el margen de acción incluye también la inacción en tanto es otra opción que se toma cuando se está frente a recursos tácticos disponibles, es decir, frente a recursos humanos, actividades y bienes.

11. González Rodríguez Juan José. Efectos perversos de las estrategias familiares en la agricultura. En: Luis Garrido Medina y Enrique Gil Calvo (eds). Estrategias Familiares. Madrid: Alianza Universidad, 1993. P. 134.

12. Ibíd. p. 17.

13. Cebotarev, Nhora. La familia como problema de investigación. En: Restrepo, Dalia (ed.). Taller de investigación aplicado al estudio de la familia. Manizales: 
Editorial Imprenta Cafetera, 1985, p. 8. Y en: Cebotarv, Nhora. Desenvolvimiento sustentável para á familia. Ponencia presentada en el Panel sobre Medio Ambiente, Congreso de Economía Doméstica. Brasil: Universidad Federal de Viscosa, Septiembre 9-11 de 1993.

14. Garrido y Gil. Op. cit., 16

15. Este aparte está basado en: DEPARTMENT FOR INTERNATIONAL DEVELOPMENT DFID, Gran Bretaña. Hojas orientativas sobre los medios de vida sostenibles. Conceptos Básicos. En línea: http://www.livelihoods.org/info/guidance_sheets_rtfs/SP-GS1.rtf Consultado el 17 octubre de 2005.

16. Chambers, Robert and Conway, R. Sustainable Rural Livelihoods: Practical Concepts for the 21st Century. IDS Discussion Paper, No 296, 1992, p. 7.

17. DEPARTMENT FOR INTERNATIONAL DEVELOPMENT DFID. Op cit.

18. Stoian Dietmar, Donovan Jason. Articulación del mundo campesino con el mercado. Integración de los enfoques Medios de Vida y Cadena Productiva. VI semana científica del CATIE, p. 14.

19. "Rural livelihood diversification is defined as the process by wich rural households construct increasingly diverse portafolio of activities and assets in order to survive and to improve their standard of living". Farrington, John et al. Op. cit.

20. Farrington, John et al. Estrategias de vida sostenibles en la práctica: Primeras aplicaciones de los conceptos en áreas rurales. Revista No 42. Junio 1999, s.p.

21. Ibíd. s.p.

22. Ellis, Frank. 2000. Rural livelihoods and Diversity in developing Countries. Oxford, University Press. New York.

23. Stoian Dietmar, Donovan Jason. Op cit.; Sadoval- Priego, Araceli Amada. Estrategias familiares de vida y su relación con desnutrición en niños menores de dos años. En: Salud Pública en México. Revista electrónica Vól. 44N0 1, Enero-Febrero de 2004. p, 41.

24. Farrington, John et al. Op.cit., s.p.

25. Mercado Mondragón Jorge. El concepto dinámicas familiares rurales de produccióny la pertinencia de su aplicación en la sociología rural. VII Congreso Latinoamericano de Desarrollo Rural. 20-24 de noviembre de 2006. Quito, Ecuador.

www.alasru.org/cdalasru2006/22\%20GT\%20Jorge\%20Mercado\%20Mondragón .pdf.Consultado el 24 de mayo de 2007. 